\title{
Pathology in Practice
}

In collaboration with the American College of Veterinary Pathologists

\section{History}

A 7-year-old 23-kg castrated male Golden Retriever was evaluated by the Ophthalmology and Oncology services at Colorado State University's Veterinary Teaching Hospital because of a 5-week history of blepharospasm, scleral injection, and epiphora from the right eye, as well as a 2-week history of progressive signs of pain and lameness in the right hind limb. The dog was undergoing chemotherapy (cyclophosphamide, hydroxydaunorubicin [doxorubicin], vincristine, and prednisone) for multicentric T-cell lymphoma that had been confirmed on the basis of results of cytologic examination of a lymph node specimen, PCR assay for antigen receptor rearrangements, and flow cytometry approximately 1.5 months earlier. At the time of the diagnosis of lymphoma, the dog had peripheral lymphadenopathy including enlarged bilateral mandibular, bilateral prescapular, and bilateral popliteal lymph nodes. Within the preceding month, the patient had a single vomiting episode and evidence of superficial pyoderma. The dog had a history of being used for hunting in New Mexico before moving to Colorado approximately 6 months prior to presentation.

\section{Clinical and Gross Findings}

Although anxious, the dog was alert and responsive. Discomfort was elicited on palpation of the right stifle joint and cervical region. Moderate effusion surrounded the stifle joint. Radiography revealed evidence of sternal lymphadenopathy and an irregular periosteal reaction along the proximomedial aspect of the right tibia suggestive of an aggressive osseous lesion. Clinicopathologic analyses revealed

Kyle Kline, DVM'; Christopher Dolan, DVM'; Brian Young, DVM'; Susan E. Lana, DVM, MS'; Linda Vap, DVM²; Richard Dubielzig, DVM ${ }^{3}$; Leandro Teixeira, DVM, MS ${ }^{3}$; Michala de Linde Henriksen, DVM, PhD'*

'Department of Clinical Sciences, College of Veterinary Medicine and Biomedical Sciences, Colorado State University, Fort Collins, CO ${ }^{2}$ Department of Microbiology, Immunology, and Pathology, College of Veterinary Medicine and Biomedical Sciences, Colorado State University, Fort Collins, CO

${ }^{3}$ Department of Pathobiology Sciences, School of Veterinary Medicine, University of Wisconsin, Madison, WI

*Corresponding author: Dr. Henriksen (michala.henriksen@colostate .edu)

https://doi.org//0.2460/javma.19.06.0305

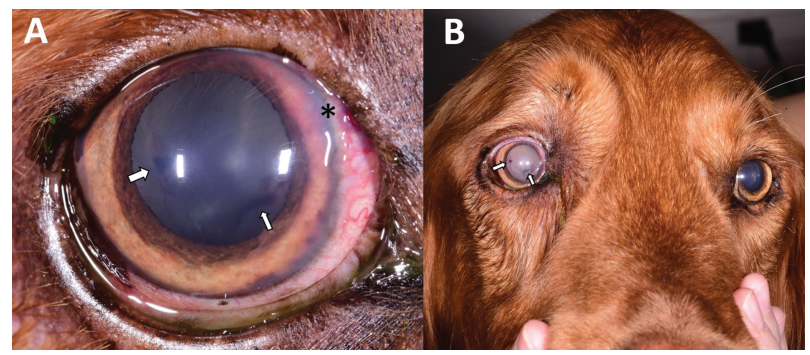

Figure I-Photographs of the right eye (A) and both eyes (B) of a 7-year-old 23-kg castrated male Golden Retriever that was undergoing chemotherapy for multicentric T-cell lymphoma diagnosed 1.5 month earlier and that had a 5-week history of blepharospasm, scleral injection, and epiphora from the right eye and a 2-week history of progressive signs of pain and lameness in the right hind limb. A-The right eye has moderate hyperemia of the conjunctiva with multiple dorsonasal areas of small, round, raised nodules at the limbus (asterisk). The pupil is mydriatic (dilated), and a complete serous retinal detachment with vitreous hemorrhage is evident (arrows). B-The appearance of the right and left eyes is distinctly different. The right eye has a mydriatic pupil, retinal detachment, and vitreous hemorrhage (arrows).

leukocytosis, mild anemia, mildly high globulin concentration, and a mildly high alkaline phosphatase. A neuro-ophthalmic examination revealed blindness of the right eye (negative menace response, dazzle reflex, and direct and consensual pupillary light reflexes) and clinically normal findings for the left eye. Ophthalmic examination of the right eye revealed episcleral injection and moderate hyperemia of the conjunctiva with multiple dorsonasal areas of small round (3- to 5-mm-diameter), raised nodules at the limbus (Figure I). The cornea was clear, and hyperemia and thickening of the iris were noted. The right eye also contained $2+$ flare (flare scale from trace to +4$)$ and cells in the aqueous humor, indicative of anterior uveitis. Fundic examination of the right eye revealed serous complete retinal detachment with a focal area of subretinal exudate in the dorsal-temporal aspect of the fundus. Ophthalmic examination of the left eye revealed clinically normal adnexa and anterior and posterior segments. Intraocular pressure of each eye was considered normal although mildly higher in the right eye $(18 \mathrm{~mm} \mathrm{Hg}$; reference range, ${ }^{1} 10$ to $25 \mathrm{~mm} \mathrm{Hg}$ ) than in the left eye (13 mm Hg).

Formulate differential diagnoses, then continue reading. 


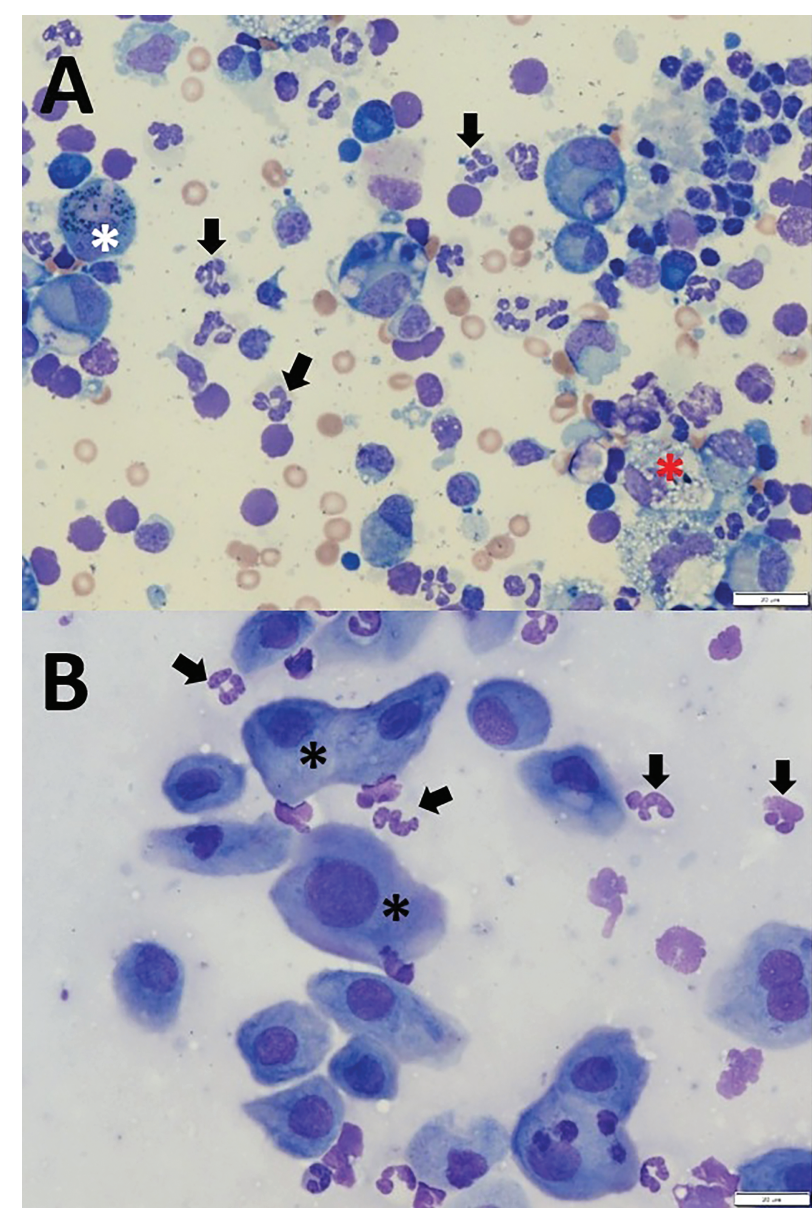

Figure 2- Cytologic images of specimens obtained from the dog's right eye. A - In a subretinal aspirate specimen, notice the mixed inflammation, composed of predominantly neutrophils (arrows) together with lymphocytes and vacuolated monocytes (red asterisk). Some of the monocytes have blue-black pigmented vacuoles in their cytoplasm, consistent with melanin (white asterisk). No infectious agents are evident. WrightGiemsa stain; bar $=20 \mu \mathrm{m}$. B-In a cytologic scrape preparation from the dorsonasal area of small round, 3- to 5-mm-diameter raised nodules at the limbus revealed suppurative inflammation (including neutrophils [arrows]) and conjunctival or corneal epithelial cells (asterisks). No microorganisms were observed, but the presence of inflammatory cells is supportive of an infectious process. Wright-Giemsa stain; bar $=20 \mu \mathrm{m}$.

\section{Clinicopathologic and Histopathologic Findings}

Subretinal centesis of the focal area of subretinal exudate was performed, and the collected fluid sample was submitted for cytologic examination. Direct smears and cytocentrifuged preparations of the subretinal fluid had evidence of nongranulomatous mixed inflammation with no detectable organisms (Figure 2). Cytologic samples of the proliferative conjunctival tissue were also collected with a cytobrush and examined; the findings were consistent with suppurative inflammation. Fungal serologic evaluation was performed. Coccidioides exposure was indicated on the basis of detection of IgG anti-Coccidioides spp

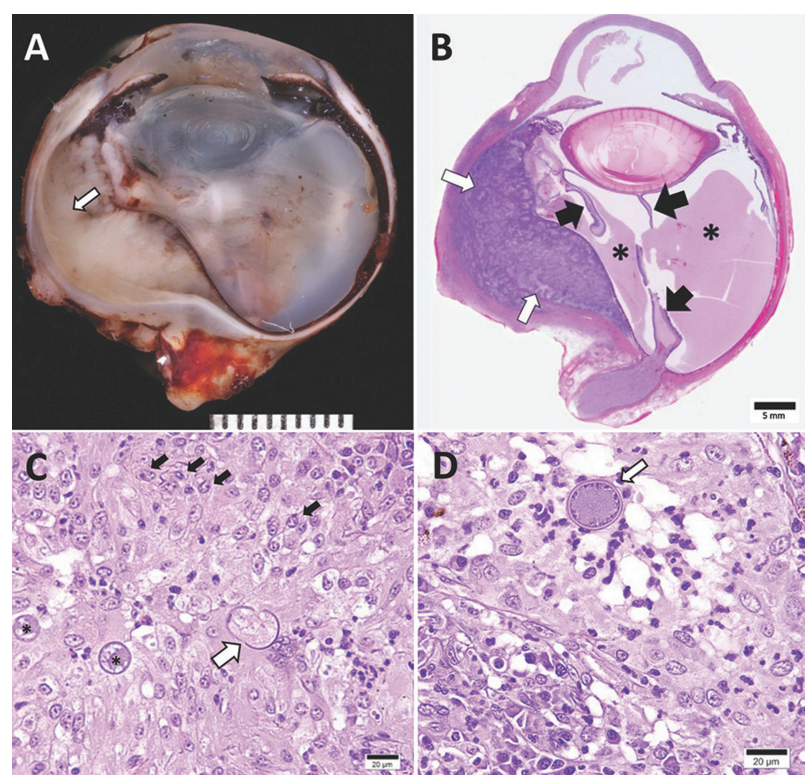

Figure 3-Images of the dog's right eye after enucleation. A-Horizontal sectioning of the formalin-fixed right globe reveals homogenous white tissue (white arrow) that infiltrates and expands the medial ciliary body, choroid, and equatorial sclera. Scale $=1 \mathrm{~cm}$. B-In a sub-gross photomicrograph of a section of the globe, notice multiple confluent pyogranulomas that have infiltrated the posterior segment of the globe (white arrows) and the detached retina (black arrows) and subretinal exudate (asterisks). H\&E stain; bar $=5 \mathrm{~mm}$. C Higher magnification view of the inflammatory infiltrate. Pyogranulomatous inflammation with neutrophils and numerous epithelioid macrophages (black arrows) surrounds intact (asterisks) and degenerated (white arrow) coccidioidal spherules. H\&E stain; bar $=20 \mu \mathrm{m}$. D-At the center of a pyogranuloma, there is a single spherule with granular to flocculent basophilic nuclear material (white arrow); the spherule is surrounded by neutrophils and epithelioid macrophages. H\&E stain; bar $=20 \mu \mathrm{m}$.

antibodies (titer, 1:32) by agar gel immunodiffusion testing. However, use of this assay for animal serum samples had not been fully characterized; for an undefined number of canine patients that do have coccidioidomycosis, the assay results indicate seronegativity, whereas subclinically infected dogs have been found to have titers as high as $1: 16 .^{2}$ Although immunosuppression associated with multicentric neoplasia and successive chemotherapy treatments has the potential to lower antibody titers in serologic evaluation of fungal infections, a titer of 1:32 is often reported to be associated with disseminated disease. ${ }^{2}$

One week later, the dog was reexamined and a diagnosis of secondary glaucoma of the right eye was made. The right eye was buphthalmic and had an intraocular pressure of $25 \mathrm{~mm} \mathrm{Hg}$ (left eye, $15 \mathrm{~mm} \mathrm{Hg}$ ). The dog's right eye was enucleated because of the grave prognosis for return of vision and comfort. The globe and retrobulbar tissue were fixed in formalin and submitted for histologic examination at the Comparative Ocular Pathology Laboratory of Wisconsin. Grossly, the medial-inferior ciliary body, choroid, and equatorial sclera were markedly infiltrated and expanded by a homogenous white tissue (Figure 3). Histologically, 
there was a predominantly pyogranulomatous inflammatory infiltrate that markedly expanded and partially replaced the ciliary body, choroid, equatorial sclera, and episclera. The inflammation was characterized by multifocal to coalescing pyogranulomas composed of a core of viable and degenerated neutrophils surrounded by several layers of epithelioid macrophages, lymphocytes, and plasma cells. Some pyogranulomas contained fungal spherules (yeasts) ranging from 16 to $40 \mu \mathrm{m}$ in diameter, each with a 1- to 3- $\mu \mathrm{m}$-thick double-contoured hyaline and refractile cell wall. Spherules contained granular to flocculent, basophilic material with rare round (3- to 5- $\mu$ m-diameter) endospores. Additionally, there was complete retinal detachment with extensive accumulation of a fibrinrich proteinaceous material containing moderate numbers of foamy macrophages, neutrophils, and scant RBCs in the subretinal space and vitreous. The detached retina had multifocal areas of hemorrhage and mild lymphocytic infiltration, but otherwise no major signs of atrophy. No signs of the previously diagnosed lymphoma were present in the ocular tissues or lesions.

\section{Morphologic Diagnosis and Case Summary}

Morphologic diagnosis and case summary: pyogranulomatous panophthalmitis with secondary glaucoma and retinal detachment caused by Coccidioides spp in a 7-year-old dog.

\section{Comments}

For the dog of the present report, the initial ophthalmic diagnosis was suspected to be an ocular manifestation of a systemic disease caused by either systemic neoplasia or infection. Neoplasia is capable of causing aggressive osseous lesions that are readily detected during radiographic evaluation. ${ }^{3}$ The dog's travel history, clinical signs, immunocompromised status as a result of chemotherapy, and radiographic findings for the right hind limb could also have been explained by systemic fungal infection (Coccidioides infection being the most common fungal infection found in New Mexico). ${ }^{4,5}$ Histopathologic detection of fungal organisms in the vitreous confirmed ocular coccidioidomycosis as the cause of this dog's serous retinal detachment with inflammatory fluid in the subretinal space. The dog was presumed to have disseminated coccidioidomycosis. The clinical, imaging, serologic, and histopathologic findings for this dog were considered sufficiently compelling to initiate systemic fluconazole administration. Chemotherapy for multicentric T-cell lymphoma was continued. The dog was alive 91 days following enucleation of the right eye.

The etiologic agent for so-called valley fever is the dimorphic fungus Coccidioides immitis, which thrives in the warm, dry climate and sandy, alkaline soils found in the western portion of the
United States. ${ }^{6}$ Infective arthroconidia (spores) from the fungus are released from disrupted soil, and their inhalation is the primary route of infection in dogs. ${ }^{7-9}$ With regard to $C$ immitis, it has been said that "Any dog that breathes air in an endemic region can become infected."10 One longitudinal study ${ }^{11}$ evaluating the relative risk of infection for dogs in regions in which Coccidioides spp are endemic advanced the notion that the risk of Coccidioides infection in dogs that spend more time outdoors or have more land to roam, such as hunting dogs, was 4.9 times as great as that in dogs that were kept indoors.

Dogs with coccidioidomycosis most commonly present with severe pulmonary signs..$^{2,10,12}$ Approximately $20 \%$ of Coccidioides infections in dogs are disseminated, ${ }^{2,12,13}$ and the most common sites of dissemination are bones, joints, and lymph nodes. ${ }^{2} \mathrm{Ocu}-$ lar manifestations of Coccidioides infection in dogs have rarely been reported ${ }^{14,15}$ and are described as uncommon in human medicine as well.6,16,17

To the authors' knowledge, only 1 case of a dog with both disseminated coccidioidomycosis and concurrent multicentric lymphoma has been documented. ${ }^{18}$ Diagnosis of coccidioidomycosis in such cases becomes a challenge because of overlapping clinical signs and the difficulty and unreliability in detection of Coccidioides spherules in aspirated tissue specimens obtained from patients. Treatment of the combination of neoplasia and fungal infection is also troublesome. Immunosuppression caused by lymphoma or systemic chemotherapy can allow the infective organisms to thrive, resulting in overwhelming dissemination of the fungus. Such immunosuppression may be the reason that the dog of the present report acquired the fungal infection and may have facilitated dissemination of the organisms to a unique location, namely an eye. In the case described in the present report, it was difficult to determine whether some of the dog's clinical signs were attributed to either lymphoma or coccidioidomycosis. Enlarged lymph nodes throughout the body, radiographic evidence of aggressive bony lesions, vomiting episodes, superficial pyoderma, and chorioretinitis with associated retinal detachment, all of which were evident in this dog, have been described in cases with disseminated fungal infection as well as with multicentric lymphoma. ${ }^{7-24}$ Without histologic examination of biopsy specimens, definitive diagnosis of the underlying cause for each of these clinical signs cannot be determined in patients with these concurrent disease processes. In some cases, a contribution from both disease processes may explain a certain clinical sign. In the 1 reported case of a canine patient with multicentric lymphoma and disseminated coccidioidomycosis, pathologists identified both neoplastic lymphocytes and Coccidioides spherules in the same lymph node. ${ }^{18}$

The case described in the present report has highlighted some important issues. Collection of information regarding an animal's travel history is imperative 
in a thorough veterinary examination. This applies to animals that currently have a diagnosed systemic disease, such as multicentric lymphoma. Serial eye examinations are warranted in animals with known coccidioidomycosis. Although rare, ocular manifestations of disseminated fungal infection in dogs develop and can induce blindness and discomfort, necessitating enucleation of the globe. Clinical signs of fungal infection may be masked by concurrent disease processes, such as multicentric lymphoma. Histologic evaluation of biopsy specimens of infected tissue often yields more definite diagnostic results than examination of cytologic samples. Cytologic findings are often nondiagnostic because of the relatively low number of spherules found in tissues. ${ }^{18}$

\section{References}

1. Gelatt KN, MacKay EO. Distribution of intraocular pressure in dogs. Vet Opbthalmol. 1998;1(2-3):109-114.

2. Graupmann-Kuzma A, Valentine BA, Shubitz LF, Dial SM Watrous B, Tornquist SJ. Coccidioidomycosis in dogs and cats: a review. J Am Anim Hosp Assoc. 2008;44(5):226-235.

3. Navarro SM, Matcuk GR, Patel DB, et al. Musculoskeletal imaging findings of hematologic malignancies. Radiographics. 2017;37(3):881-900.

4. Brown J, Benedict K, Park BJ, Thompson GR III. Coccidioidomycosis: epidemiology. Clin Epidemiol. 2013;5:185-197.

5. Guarner J, Brandt ME. Histopathologic diagnosis of fungal infections in the 21st century. Clin Microbiol Rev. 2011;24(2):247-280.

6. Ventocilla M. Ocular Coccidioidomycosis. Medscape; 2015.

7. Fisher MC, Koenig GL, White TJ, et al. Molecular and phenotypic description of Coccidioides posadasii sp. nov., previously recognized as the non-California population of Coccidioides immitis. Mycologia. 2002;94: 73-84.

8. Barsanti JA, Kenneth JL. Coccidioidomycosis. In: Greene CE, ed. Clinical Microbiology and Infectious Diseases of the Dog and Cat. Elsevier. 1984;710-721.
9. Greene RT. Coccidioidomycosis. In: Greene CE, ed. 2nd ed. Clinical Microbiology and Infectious Diseases of the Dog and Cat. Elsevier. 1998;391-398.

10. Rubensohn M, Stack S. Coccidioidomycosis in a dog. Can Vet J. 2003;44(2):159-160.

11. Butkiewicz CD, Shubitz LE, Dial SM. Risk factors associated with Coccidioides infection in dogs. J Am Vet Med Assoc. 2005;226(11):1851-1854.

12. Davidson AP, Pappagianis D. Canine coccidioidomycosis: 1970-1993. Proceed 5th Int Conf Coccidioidomycosis, Stanford. 1994;551-162.

13. Greene RT, Troy GC. Coccidioidomycosis in 48 cats: a retrospective study (1984-1993). J Vet Intern Med. 1995;9: 86-91.

14. Angell JA, Merideth RE, Shively JN, Sigler RL. Ocular lesions associated with coccidioidomycosis in dogs: 35 cases (1980-1985). J Am Vet Med Assoc. 1987;190(10):1319-1322.

15. Shively JN, Whiteman CE. Ocular lesions in disseminated coccidioidomycosis in 2 dogs. Pathol Vet. 1970;7(1):1-6.

16. Rodenbiker HT, Ganley JP. Ocular coccidioidomycosis. Surv Ophthalmol. 1980;24(5):263-290.

17. Blumenkranz MS, Stevens DA. Endogenous coccidioidal endophthalmitis. Opbthalmology. 1980;87(10):974-984

18. Jeroski A. Multicentric lymphoma and disseminated coccidioidomycosis in a dog. Can Vet J. 2003;44(1):62-64.

19. Millman TM, O'Brien TR, Suiter PF, Wolf AM. Coccidioidomycosis in the dog: it's a radiographic diagnosis. Vet Radiol Ultrasound. 1979;20(2):50-65.

20. Oldfield EC III, Olson PE, Bone WD, Bradshaw DA, Martin CR. Coccidioidomycosis presenting as neoplasia: another great imitator disease. Infect Dis Clin Pract. 1995;4:87-92.

21. Ramírez-Romero R, Silva-Pérez RA, Lara-Arias J, et al. Coccidioidomycosis in biopsies with presumptive diagnosis of malignancy in dogs: report of three cases and comparative discussion of published reports. Mycopatbologia. 2016;181:151-157.

22. Shubitz LF, Dial SM. Coccidioidomycosis: a diagnostic challenge. Clin Tech Small Anim Pract. 2005;20(4):220-226.

23. Arora NP, Taneja V, Reyessacin C, Bhanot R, Natesan SK Coccidioidomycosis masquerading as malignancy. BMJ Case Rep. 2012: 2012: bcr1220115357.

24. Crum NF. Disseminated coccidioidomycosis with cutaneous lesions clinically mimicking mycosis fungoides. Int J Dermatol. 2005;44(11):958-960. 